\title{
Error Rates for Hybrid SC/MRC Systems on Nakagami-m Channels
}

\author{
A. Annamalai ${ }^{1}$ and C. Tellambura ${ }^{2}$ \\ 1. Bradley Department of Electrical and Computer Engineering, Virginia Tech, 206 N. Washington St., Suite 400 \\ Alexandria, VA 22314, USA, Tel:+1-703-535-3459, Fax:+1-703-518-8085, E-mail: annamalai@vt.edu \\ 2. School of Computer Science and Software Engineering, Monash University, Clayton, Victoria 3168, Australia \\ Tel: +1-613-9905-3296, Fax: +1-613-9905-3402, E-mail: chintha@dgs.monash.edu.au
}

\begin{abstract}
The efficacy of an hybrid $M / L-S C / M R C$ receiver structure (also known as generalized selection combining) in a variety of fading environments is analyzed by deriving considerably simpler expressions for the statistics (i.e., moment generating function (MGF) and cumulative distribution function (CDF)) of the combiner output signal-to-noise ratio (SNR) on Nakagami-m channels with arbitrary parameters. Different from previous studies, these results hold for arbitrary orders of $M$ and $L$ as well as for any real values of fading severity index $m \geq 0.5$. A simple procedure for deriving an exact closed-form expression for the MGF of SNR when the fading index assumes a positive integer $m$ value is also outlined. These MGFs are then used to derive the average symbol error probability (ASEP) for a broad class of binary and M-ary modulations employing coherent SC/MRC receiver. Analytical expressions for computing the outage rate of error probability and the average combined output SNR are also derived. Finally, computationally efficient but approximate solutions for the MGF of SNR are presented.
\end{abstract}

\section{INTRODUCTION}

Hybrid SC/MRC diversity scheme has received considerable attention in the literature owing to its ability to counter balance the detrimental effects of deep fades on wireless channels, while achieving a good compromise between the receiver performance and the implementation complexity (fewer electronics as well as lower power consumption) [1]-[7]. In an $M / L$-SC/MRC system, $M$ strongest diversity branches out of a total of $L$ diversity branches are first selected and then they are coherently combined in a MRC combiner to produce the decision statistic. The study of hybrid SC/MRC receiver is important both from a practical viewpoint and theoretical standpoint because this model encapsulates both $\mathrm{SC}(M=1)$ and $\mathrm{MRC}(M=L)$ receiver performance as limiting cases. Also, in some situations such as in wideband CDMA applications, the number of available correlator resources limits the number of multipaths that can be utilized in a typical rake combiner. Obviously, in such a scenario $M / L-S C / M R C$ receiver merits consideration because it outperforms $M$-MRC (i.e., signal combination from the first $M$ fingers) receiver configuration owing to the improved SNR statistic at the output of the hybrid combiner [3]. It is also attractive for reduced complexity implementation of antenna array in millimeter wave communications [6].

Most of the previous studies with the exception of [3]-[5] only examines the performance of $\mathrm{SC} / \mathrm{MRC}$ in Rayleigh fading. In [3], the analysis proceeds in similar manner to that of put forward by [1] and therefore, the final result involves the computation of multiple integrals (which is not desirable for larger values of $M$ ). In [4], the performance of 2/3-SC/MRC and 2/4-SC/MRC are examined over Nakagami-m fading channels. As in [3], the results are also limited to BPSK modulation. Besides, the final expressions require the evaluation of multiple infinite series (i.e., $(L-2)$-fold infinite series even for the specific case of $M=2$ ) and their method is not amenable to analysis for any $M>2$ or other modulation formats such as MPSK, MQAM, DQPSK, to name a few. In [5], the authors applied the Dirichlet transformation to simply certain multiple integrals that arise in the distribution theory of ordered Gamma random variables (assuming positive integer fading index) and subsequently outlined a procedure for obtaining the ASEP suitable for numerical computation. Their expression for the MGF [5, Eq. (15)] for non-integer $m$ will require the evaluation of $(L-1)$-fold nested integral, which can be tedious and complicated, particularly for large $L$ values. Even for the positive integer $m$ case, one have to simplify the $(L-1)$-fold integral for the MGF for each different $M$ and/or $L$ values. As such, this approach does not lend itself to the analysis of average combined output SNR. By contrast, in this paper we first derive a considerably simpler expression for the MGF of SNR at the output of hybrid $M / L-\mathrm{SC} / \mathrm{MRC}$ combiner. Different from [5], our initial formulation of the above ordered statistics problem will require evaluation of an $M$-fold integral for the MGF and this expression holds for arbitrary $L$. Further simplifications of this integral are possible and they are also discussed in this paper. This MGF is then used to derive ASEP for a broad range of modulation formats (including CPSK, CFSK, DPSK, M-ary square QAM, star-QAM, arbitrary two-dimensional signal constellations, MPSK, MDPSK, DQPSK with Gray coding and MFSK) employing $\mathrm{SC} / \mathrm{MRC}$ receiver. It is also important to note that the MGF expression also applies to the performance evaluation of $\mathrm{SC} / \mathrm{EGC}$ quadratic receiver.

\section{EXACT ANALYSIS}

In this section, we first derive the hybrid combiner output statistics by modelling the branch amplitudes as i.i.d. Nakagami-m random variables and then proceed with their applications to develop concise analytical expressions to 
compute the ASEP and outage probability for different modulation schemes and to determine the mean combined output SNR.

From [8], we know that the joint probability density function (PDF) of selecting $M$ strongest diversity branches from a total of $L$ available diversity branches is given by

$$
p_{\gamma_{1}, \ldots, \gamma_{\lambda}}\left(\gamma_{1}, \ldots, \gamma_{M}\right)=M !\left(\begin{array}{c}
L \\
M
\end{array}\right)\left[F\left(\gamma_{M}\right)\right]^{L-1 /} \prod_{i=1}^{M} p\left(\gamma_{1}\right)
$$

where $\gamma_{1} \geq \ldots \geq \gamma_{M} \geq 0, p($.$) and F($.) correspond to the PDF and CDF of SNR for a single diversity branch, respectively. Recognizing that the MGF of combiner output SNR $\phi_{y}($.$) is$ the key to unified analysis of a wide range of modulation schemes over wireless channels, in the following we will immediately derive the desired MGF. Let $\gamma=\sum_{i=1}^{\prime \prime} \gamma_{l}$ denote the combiner output SNR, and therefore we have

$$
\begin{aligned}
& \phi_{y}(s)=\int_{0}^{\infty} e^{-s \gamma_{1}} \int_{0}^{\gamma_{1}} e^{-s \gamma_{2}} \ldots \int_{0}^{\gamma_{M-1}} e^{-s \gamma_{M}} p_{\gamma_{1}}, \ldots, \gamma_{N j}\left(\gamma_{1}, \ldots, \gamma_{M}\right) d \gamma_{M} \ldots d \gamma_{2} d \gamma_{1} \\
& =M !\left(\begin{array}{l}
L \\
M
\end{array}\right) \int_{0}^{\infty} e^{-s \gamma_{1}} p\left(\gamma_{1}\right) \int_{0}^{\gamma_{1}} e^{-\alpha \gamma_{2}} p\left(\gamma_{2}\right) \ldots \int_{0}^{\gamma_{H N-2}} e^{-s \gamma_{M-1}} p\left(\gamma_{M-1}\right) \\
& \times \int_{0}^{\gamma_{M S-1}} e^{-\gamma_{\gamma_{A}}} p\left(\gamma_{M}\right)\left[F\left(\gamma_{M}\right)\right]^{L-. .1} d \gamma_{M} \ldots d \gamma_{2} d \gamma_{1}
\end{aligned}
$$

where $\gamma_{0}=\infty$,

$$
\begin{gathered}
p(x)=\frac{1}{\Gamma(m)}\left(\frac{m}{\bar{\gamma}}\right)^{m} x^{m-1} e^{-m \cdot x / \bar{\gamma}}, \\
F(x)=\frac{\gamma\left(m, \frac{m x}{\bar{\gamma}}\right)}{\Gamma(m)}=\left\{\begin{array}{l}
\frac{1}{\Gamma(m)} \sum_{n=0}^{\infty} \frac{(-1)^{n}}{n !(m+n)}\left(\frac{m x}{\bar{\gamma}}\right)^{m+n}, \text { real } m \geq \frac{1}{2} \\
1-e^{-m x / \bar{\gamma}} \sum_{n=0}^{m-1} \frac{1}{n !}\left(\frac{m x}{\bar{\gamma}}\right)^{n}, \text { positive integer } m
\end{array}\right.
\end{gathered}
$$

and $\bar{\gamma}$ is the average SNR/symbol per branch.

Careful examination of (2) in conjunction with (3) and (4) reveals several interesting results: (a) If the fading amplitudes follow Rayleigh or Nakagami-m distribution with positive integer $m$, the $M$-fold nested integral can be evaluated in closed form using identities [9, Eq. (3.351.1)] and [9, Eq. (3.351.3)]; (b) For Nakagami-m fading with real $m$, an infinite series solution can be readily obtained with the aid of [9, Eq. (3.381.1)] and [9, Eq. (6.455.2)]. The resultant expression is considerably simpler then [4] and more importantly, it holds for arbitrary $M$ and $L$ values; (c) Eq. (2) removes the inherent disadvantage of the Dirichlet transformation technique particularly for large values of $L$ since it only involves the evaluation of $M$-fold integral instead of $(L-1)$-fold integral. Furthermore, it circumvents the need to perform simplifications of the nested integral for each different $L$ for a specified $M$. At this juncture, it is also worth highlighting that (2) holds for other common fading distributions (e.g., Rician and Nakagami-Hoyt) provided all the diversity branches are independent and identically distributed. Next, we will consider the simplifications of (2) for Nakagami-m fading with positive integer fading index and the real valued fading index cases explicitly.

\section{A. Real Valued Fading Severity Index}

In order to simplify (2), we first exploit identity [9, Eq $(0.314)]$ to rewrite $[F(x)]^{v}$ ( $v$ is a natural number) as

$$
[F(x)]^{v}=\left[\frac{(m / \bar{\gamma})^{\prime \prime \prime}}{\Gamma(m)}\right]^{\nu} \sum_{n=0}^{\infty} c_{n} x^{n+m v}
$$

where $a_{n}=\frac{(-m / \bar{\gamma})^{n}}{n !(m+n)}, c_{0}=a_{0}^{v}=m^{-\nu}$ and

$$
c_{t}=\sum_{n=1}^{\prime}\left(\frac{n v-t+n}{t a_{0}}\right) a_{n} c_{t-n}=\frac{m}{t} \sum_{n=1}^{\prime} \frac{(n v-t+n)}{n !(m+n)}\left(\frac{-m}{\bar{\gamma}}\right)^{n} c_{t-n}, t \geq 1 \text {. }
$$

Next, substituting (5) into (2), we obtain

$$
\phi_{\gamma}(s)=M !\left(\begin{array}{l}
L \\
M
\end{array}\right)\left[\frac{(m / \bar{\gamma})^{m}}{\Gamma(m)}\right]^{L} \sum_{n=0}^{\infty} c_{n} G(m, m / \bar{\gamma}, n, M)
$$

where

$$
\begin{aligned}
& G(m, \lambda, n, M)=\int_{0}^{\infty} e^{-\gamma_{1}(x+\lambda)} \gamma_{1}^{m-1} \int_{0}^{\gamma_{1}} e^{-\gamma_{2}(x+\lambda)} \gamma_{2}^{m-1} \ldots \int_{0}^{\gamma_{M-2}} e^{-\gamma_{M-1}(x+\lambda)} \gamma_{M-1}^{m-1} \\
& \times \int_{0}^{\gamma_{A}+1} e^{-\gamma_{A}(s+\lambda)} \gamma_{M}^{\prime \prime+m(L-1 /+1)-1} d \gamma_{A_{1}} \ldots d \gamma_{2} d \gamma_{1}
\end{aligned}
$$

It is apparent that if we can find an analytical solution to the nested integral $G(m, \lambda, n, M)$ depicted in (7), then we can achieve our goal, namely to obtain a simple analytical solution for (2) which holds for arbitrary order of $L$. Fortunately, this integral can be evaluated without great difficulty and the results are summarized below for different values of $M$ :

$$
\begin{gathered}
G(m, \lambda, n, 1)=\frac{\Gamma(n+m L)}{(s+\lambda)^{n+m L}} \\
G(m, \lambda, n, 2)=\frac{\Gamma(n+m L)_{2} F_{1}\left[1, n+m L ; n+m(L-1)+1 ; \frac{1}{2}\right]}{[n+m(L-1)][2(s+\lambda)]^{n+m L}}(9) \\
G(m, \lambda, n, 3)=\sum_{n_{1}=0}^{\infty} \frac{(-1 / 2)^{n_{1}} \Gamma\left(n_{1}+n+m L\right)}{n_{1} !\left[n+n_{1}+m(L-2)\right]\left[n+n_{1}+m(L-1)\right]} \\
\quad \times \frac{{ }_{2} F_{1}\left[1, n_{1}+n+m L ; n_{1}+n+m(L-1)+1 ; \frac{1}{2}\right]}{[2(s+\lambda)]^{n+m L}} \\
G(m, \lambda, n, 4)=\frac{1}{[2(s+\lambda)]^{n+m L} \sum_{n_{1}=0}^{\infty} \frac{(-1 / 2)^{n_{1}}}{n_{1} !\left[n+n_{1}+m(L-3)\right]}} \\
\times \sum_{n_{2}=0}^{\infty} \frac{(-1 / 2)^{n_{2}} \Gamma\left(n+n_{1}+n_{2}+m L\right)}{n_{2} !\left[n+n_{1}+n_{2}+m(L-2)\right]\left[n+n_{1}+n_{2}+m(L-1)\right]} \\
\times{ }_{2} F_{1}\left[1, n_{1}+n_{2}+n+m L ; n_{1}+n_{2}+n+m(L-1)+1 ; \frac{1}{2}\right]
\end{gathered}
$$

To obtain the above results, we have utilized the following three integral identities and an infinite series representation for the incomplete Gamma function:

$$
\begin{gathered}
\int_{0}^{\infty} t^{a-1} e^{-h t} d t=\Gamma(a) / b^{a} \\
\int_{0}^{z} t^{a-1} e^{-h t} d t=\gamma(a, b z) / b^{a} \\
\int_{0}^{\infty} t^{\mu-1} e^{-\beta 1} \gamma(\nu, \alpha t) d t=\frac{\alpha^{v} \Gamma(\mu+v)}{v(\alpha+\beta)^{\mu+v^{2}}} F_{1}\left[1, \mu+v ; v+1 ; \frac{\alpha}{\alpha+\beta}\right] \\
\gamma(a, b z)=\sum_{n=0}^{\infty} \frac{(-1)^{\prime \prime}}{n !(a+n)}(b z)^{a+n}
\end{gathered}
$$

From the above developments, one may observe that the $M$-fold nested integral $G(m, \lambda, n, M)$ for $M \geq 3$ may be com- 
puted using ( $M-2)$-fold infinite series, viz.,

$$
\begin{aligned}
& G(m, \lambda, n, M)=\frac{1}{[2(s+\lambda)]^{n+m L}} \sum_{n_{1}=0}^{\infty} \frac{(-1 / 2)^{n_{1}}}{n_{1} !\left[n+m(L-M+1)+n_{1}\right]} \\
& \times \sum_{n_{2}=0}^{\infty} \frac{(-1 / 2)^{n_{2}}}{n_{2} !\left[n+m(L-M+2)+n_{1}+n_{2}\right]} \times \ldots \\
& \times \sum_{n_{M-3}=0}^{\infty} \frac{(-1 / 2)^{M_{H-3}}}{n_{H L-3} !\left[n+m(L-3)+\sum_{y=1}^{M-3} n_{y}\right]} \\
& \times \sum_{n_{M-2}=0}^{\infty} \frac{(-1 / 2)^{M_{M-2}} \Gamma\left(n+m L+\sum_{\nu=1}^{M-2} n_{y}\right)}{n_{M-2} !\left[n+m(L-2)+\sum_{y=1}^{M-2} n_{y}\right]\left[n+m(L-1)+\sum_{y=1}^{M-2} n_{y}\right]} \\
& \times{ }_{2} F_{1}\left[1, n+m L+\sum_{y=1}^{M-2} n_{y} ; n+m(L-1)+1+\sum_{y=1}^{M-2} n_{y} ; \frac{1}{2}\right]
\end{aligned}
$$

By invoking Gil-Pelaez's inversion theorem (which gives a relation between the $\mathrm{CDF}$ and MGF of a random variable) and then using trapezoidal rule approximation, we get a numerically efficient formula (i.e., rapidly converging series) for calculating the CDF of hybrid SC/MRC combiner output SNR:

$$
\begin{aligned}
F_{\gamma}(x) & =\frac{1}{2}-\frac{1}{\pi} \int_{0}^{\infty} \frac{1}{\omega} \operatorname{Imag}\left[\phi_{\gamma}(-j \omega) \exp (-j \omega x)\right] d \omega \\
& \cong \frac{1}{2}-\frac{2}{\pi} \sum_{\substack{n=1 \\
n \text { udd }}}^{\infty} \frac{1}{n} \operatorname{Imag}\left[\phi_{\gamma}\left(\frac{-j 2 \pi n}{T}\right) \exp \left(\frac{-j 2 \pi n x}{T}\right)\right]
\end{aligned}
$$

where coefficient $T$ is selected sufficiently large such that $\operatorname{Pr}(x>T) \leq \varepsilon$, and $\varepsilon$ can be set to a very small value. To the best of our knowledge, analytical expressions for computing the CDF of SC/MRC output SNR have been restricted to Rayleigh fading only in the literature and there is no result available for Nakagami-m fading.

The applications of the MGF and CDF expressions for characterizing the performance of SC/MRC diversity systems in terms of ASEP, outage probability and the mean combined SNR are briefly discussed below.

\section{A.I ASEP Analysis}

Let us first consider coherent BPSK and BFSK modulation schemes. Using a desired exponential integral representation for the complementary incomplete Gamma function [12] or complementary error function, the ASEP can be readily shown as

$$
\bar{P}_{b}=\frac{1}{\pi} \int_{0}^{\pi / 2} \phi_{r}\left(\frac{a}{\cos ^{2} \theta}\right) d \theta
$$

where $a=1$ for BPSK and $a=1 / 2$ for BFSK, and $\phi_{y}($.$) is$ defined in (6). The above integral can be further simplified using integral identity

$$
\begin{gathered}
\int_{0}^{\pi / 2} \frac{1}{(\tan \theta)^{2 v-1}}\left(\frac{\cos ^{2} \theta}{\beta \cos ^{2} \theta+\alpha}\right)^{\mu} d \theta=\frac{\Gamma(1-v) \alpha^{\nu} \Gamma(\mu+v)}{2 \Gamma(\mu+1)(\alpha+\beta)^{\mu+v}} \\
\quad \times{ }_{2} F_{1}\left[1, \mu+v ; \mu+1 ; \frac{\beta}{\alpha+\beta}\right] \text { for } 0<v<1
\end{gathered}
$$

which reduces to

$$
\begin{aligned}
I(\alpha, \beta, \mu) & =\int_{0}^{\pi / 2}\left(\beta+\frac{\alpha}{\cos ^{2} \theta}\right)^{-\mu} d \theta \\
& =\frac{\sqrt{\pi \alpha}{ }_{2} F_{1}\left[1, \mu+\frac{1}{2} ; \mu+1 ; \frac{\beta}{\alpha+\beta}\right] \Gamma(\mu+1 / 2)}{2 \Gamma(\mu+1)(\alpha+\beta)^{\mu+\frac{1}{2}}}
\end{aligned}
$$

when $v=1 / 2$, which is exactly the identity needed in our case. For instance, (14) reduces to (17) and (18) for $M=2$ and $M=3$ cases, respectively:

$$
\begin{aligned}
\bar{P}_{b}= & \frac{2}{\pi}\left(\begin{array}{l}
L \\
2
\end{array}\right)\left[\frac{(m / \bar{\gamma})^{m}}{\Gamma(m)}\right]^{L} \sum_{n=0}^{\infty} c_{n} \frac{\Gamma(n+m L)}{[n+m(L-1)] 2^{n+m L}} \\
& \times{ }_{2} F_{1}\left[1, n+m L ; n+m(L-1)+1 ; \frac{1}{2}\right] I\left(a, \frac{m}{\bar{\gamma}}, n+m L\right)(17) \\
\bar{P}_{b}= & \frac{1}{\pi} M !\left(\begin{array}{l}
L \\
M
\end{array}\right)\left[\frac{(m / \bar{\gamma})^{m}}{\Gamma(m)}\right]^{L} \sum_{n=0}^{\infty} \frac{c_{n}}{2^{n+m L}} \sum_{n_{1}=0}^{\infty} \frac{(-1 / 2)^{n_{1}}}{n_{1} !\left[n+n_{1}+m(L-2)\right]} \\
& \times \frac{\Gamma\left(n_{1}+n+m L\right)}{\left[n+n_{1}+m(L-1)\right]} I\left(a, \frac{m}{\bar{\gamma}}, n+m L\right) \\
& \times{ }_{2} F_{1}\left[1, n_{1}+n+m L ; n_{1}+n+m(L-1)+1 ; \frac{1}{2}\right]
\end{aligned}
$$

As a check, we find that (17) agrees with [4, Eq. (11)] for the special case of $2 / 3-\mathrm{SC} / \mathrm{MRC}$ receiver.

It is evident that for any $M \geq 2$, our final solution for the ASEP (which holds for arbitrary order of $L$ ) can be written as $(M-1)$ order infinite sum whose individual terms will involve a product of two Gauss hypergeometric functions. The above development is interesting since [4] concluded their work by stating that it is not possible to derive an infinite series solution for the ASEP for any $M>2$. Even for $M=2$, their solution will require the computation of $(L-2)$ order infinite sum whose individual terms will involve a product of two Gauss hypergeometric functions. Whereas in our case, it only requires the evaluation of a single infinite series!

We would like to emphasize that the ASEP for many other modulation schemes that employs $\mathrm{SC} / \mathrm{MRC}$ receiver can also be expressed in terms of a single finite-range integral with the integrand composed of MGF $\phi_{y}($.$) similar to (14).$ For example, the ASEP for arbitrary two-dimensional signal constellations may be computed using

$$
\bar{P}_{s}=\frac{1}{2 \pi} \sum_{k=1}^{s} W_{k} \int_{0}^{n_{k}} \phi_{r}\left[\frac{a_{k} \sin ^{2}\left(\varphi_{k}\right)}{\sin ^{2}\left(\theta+\varphi_{k}\right)}\right] d \theta
$$

where $S$ is the total number of signal points or decision subregions, $W_{k}$ is the a priori probability of the symbol to which subregion $k$ corresponds, $a_{k}, \eta_{k}$ and $\varphi_{k}$ are coefficients (constants) relating to decision subregion $k$. For our subsequent developments, it is more convenient to rewrite (19) as

$$
\begin{aligned}
\bar{P}_{x}= & M !\left(\begin{array}{c}
L \\
M
\end{array}\right)\left[\frac{(m / \bar{\gamma})^{m}}{\Gamma(m)}\right]^{L} \sum_{n=0}^{\infty} c_{n} \tilde{G}(m, n, M) \\
& \times \frac{1}{2 \pi} \sum_{k=1}^{S} W_{k} \int_{\varphi_{q_{k}}}^{\eta_{k}+\varphi_{k}}\left[\frac{a_{k} \sin ^{2}\left(\varphi_{k}\right)}{\sin ^{2}(\theta)}+\frac{m}{\bar{\gamma}}\right]^{-(n+m L)} d \theta
\end{aligned}
$$

where $\tilde{G}(m, n, M)=G(m, \lambda, n, M)(s+\lambda)^{n+m L}$ (and therefore $\tilde{G}(m, n, M)$ is independent of variable $s)$. It is interesting to 
note that the trigonometric integral in (20) can also be evaluated in closed-form if $m L$ assumes a positive integer value [10]. For the sake of brevity, we have omitted the derivations for other common modulation schemes such as MPSK, MDPSK, MQAM, DQPSK, star-QAM and so on. Each of these cases can be treated in a straight-forward fashion by utilizing the results found in [10]-[12].

\section{A. 2 Average Combined SNR}

Average combined SNR is another useful performance measure of diversity systems. Since the average combined SNR $\bar{\gamma}_{g s c}$ is the first moment (mean) of the random variable $\gamma$, it can be determined by differentiating the MGF (6) with respect to $s$ and then evaluating the derivative at $s=0$ :

$$
\begin{aligned}
\bar{\gamma}_{g: c} & =-\left.\frac{\mathrm{d}}{\mathrm{d} s} \phi_{\gamma}(s)\right|_{s=0} \\
& =\frac{M !}{[\Gamma(m)]^{L}}\left(\begin{array}{c}
L \\
M
\end{array}\right) \sum_{n=0}^{\infty} c_{n} \tilde{G}(m, n, M)(n+m L)\left(\frac{\bar{\gamma}}{m}\right)^{n+1}
\end{aligned}
$$

To the best of our knowledge, the above expression is new. Notice also that the above expression applies for any orders of $M$ and/or $L$ as well as for arbitrary fading index.

\section{A.3 Outage Probability}

The outage rate of error probability $P_{v u t}$ is defined as the probability that the instantaneous symbol error probability of the system exceeds a specified value (say $P_{*}$ ). This is equivalent to calculating the probability that the combiner output SNR falls below a predetermined threshold $\gamma^{*}$ (a coefficient that is dependent on the modulation type), viz.,

$$
P_{o u t}=\int_{0}^{*} p_{\gamma}(x) d x=F_{\mathrm{y}}\left(\gamma^{*}\right)
$$

which is essentially the CDF of SNR evaluated at $\gamma^{*}$. Consequently, (13) (in conjunction with (6)) can be used to facilitate the outage analysis. The threshold $\gamma^{*}$ can be obtained by solving $P_{s}\left(\gamma^{*}\right)=P_{t}$, where $P_{s}($.$) corresponds$ to the symbol error rate in an AWG channel. Closed-form solution for $\gamma^{*}$ is available for several common modulation schemes and they are summarized in Table 1 of [11].

\section{B. Positive Integer Fading Severity Index}

Similar to the development for the real fading index case in subsection IIA, we first rewrite $[F(x)]^{\nu}$ as

$$
\begin{aligned}
{[F(x)]^{\nu} } & =\sum_{k=0}^{\nu}\left(\begin{array}{l}
\nu \\
k
\end{array}\right)\left[-\sum_{z=0}^{m-1} \frac{1}{z !}\left(\frac{m x}{\bar{\gamma}}\right)^{z}\right]^{k} e^{-m k x / \bar{\gamma}} \\
& =\sum_{k=0}^{\nu}(-1)^{k}\left(\begin{array}{l}
v \\
k
\end{array}\right) e^{-m k x / \bar{\gamma}} \sum_{z=0}^{k(m-1)} \beta(z, k, m)\left(\frac{m x}{\bar{\gamma}}\right)^{z}
\end{aligned}
$$

by expanding the power term binomially and then utilize multinomial theorem to calculate the coefficients $\beta(., \ldots,$.$) :$

$$
\begin{aligned}
\beta(q, n, c) & =\sum_{i=q-c+1}^{4} \frac{\beta(i, n-1, c)}{(q-i) !} I_{[0,(n-1)(c-1)]}(i) \\
\text { where } I_{[a, b]}(i) & =\left\{\begin{array}{ll}
1 & a \leq i \leq b \\
0 & \text { otherwise }
\end{array}, \beta(0,0, c)=\beta(0, n, c)=1,\right.
\end{aligned}
$$$$
\beta(q, 1, c)=1 / q \text { ! and } \beta(1, n, c)=n \text {. }
$$

Using (23) in (2), the MGF of $M / L-S C / M R C$ combiner output SNR may be simplified as

$$
\begin{aligned}
\phi_{y}(s)= & \frac{M !}{[\Gamma(m)]^{M}}\left(\begin{array}{l}
L \\
M
\end{array}\right) \sum_{k=0}^{L-A I}(-1)^{k}\left(\begin{array}{c}
L-M \\
k
\end{array}\right) \sum_{z=0}^{k(m-1)} \beta(z, k, m) \\
& \times\left(\frac{m}{\bar{\gamma}}\right)^{m A L+z} F(m / \bar{\gamma}, m, z, k, M)
\end{aligned}
$$

where

$$
\begin{gathered}
F(\lambda, m, z, k, M)=\int_{0}^{\infty} e^{-\gamma_{1}(s+\lambda)} \gamma_{1}^{m-1} \int_{0}^{\gamma_{1}} e^{-\gamma_{2}(x+\lambda)} \gamma_{2}^{m-1} \ldots \int_{0-2}^{\gamma_{A-2}} e^{-\gamma_{A-1}(s+\lambda)} \gamma_{M-1}^{m-1} \\
\times \int_{0}^{\gamma_{M-1}} e^{-\gamma_{M}\left(x+\lambda_{1}+\lambda k\right)} \gamma_{M i}^{z+m-1} d \gamma_{M} \ldots d \gamma_{2} d \gamma_{1}
\end{gathered}
$$

and $\gamma_{0}=\infty$

Once again, our task reduces to finding a simple analytical solution to the nested integral $F(\lambda, m, z, k, M)$. Although (6) (in conjunction with (12)) encapsulates the positive integer fading index case, but the computation of $\phi_{\gamma}($.$) will involve$ $(M-2)$ order infinite sum. By contrast, here we show that it is possible to derive a closed-form expression for the MGF $\phi_{y}($.$) when m$ assumes a positive integer value since (26) can be evaluated in closed-form for a specified $M$. It should be emphasized that our expression holds for arbitrary order of $L$ and the simplifications of $F(\lambda, m, z, k, M)$ ( $M$-fold integral) only need to be carried out once for each different values of $M$. These results, for several practical values of $M$, are summarized below:

$$
\begin{aligned}
& F(\lambda, m, z, k, 1)=\frac{\Gamma(z+m)}{(s+\lambda+\lambda k)^{z+m}} \\
& F(\lambda, m, z, k, 2)=\frac{\Gamma(z+m)}{(s+\lambda+\lambda k)^{z+m}}\left[\frac{\Gamma(m)}{(s+\lambda)^{m}}-\sum_{n_{1}=0}^{z+m-1} \frac{(s+\lambda+k \lambda)^{n_{1}}}{n_{1} !}\right. \\
& \left.\times \frac{\Gamma\left(n_{1}+m\right)}{(2 s+2 \lambda+\lambda k)^{n_{1}+m}}\right] \\
& F(\lambda, m, z, k, 3)=\frac{\Gamma(z+m)}{(s+\lambda+\lambda k)^{z+m}}\left[\frac{\Gamma(m)}{(s+\lambda)^{2 m}}(\Gamma(m)\right. \\
& \left.-\sum_{n_{2}=0}^{m-1} \frac{\Gamma\left(n_{2}+m\right)}{n_{2} ! 2^{n_{2}+m}}\right)-\sum_{n_{1}=0}^{2+m-1} \frac{(s+\lambda+k \lambda)^{n_{1}} \Gamma\left(n_{1}+m\right)}{n_{1} !(2 s+2 \lambda+\lambda k)^{n_{1}+m}}\left(\frac{\Gamma(m)}{(s+\lambda)^{m}}\right. \\
& \left.\left.-\sum_{n_{2}=0}^{n_{1}+m-1} \frac{(2 s+2 \lambda+k \lambda)^{n_{2}} \Gamma\left(n_{2}+m\right)}{n_{2} !(3 s+3 \lambda+\lambda k)^{n_{2}+m}}\right)\right]
\end{aligned}
$$

Closed-form solutions for other values of $M$ can be derived in a similar fashion. To obtain the these results, we need to simplify (26) with the aid of only the two integral identities listed below:

$$
\begin{gathered}
\int_{0}^{\infty} t^{n-1} e^{-h t} d t=(n-1) ! / b^{n} \\
\int_{0}^{2} t^{n-1} e^{-h t} d t=\frac{\gamma(n, b z)}{b^{n}}=\frac{(n-1) !}{b^{n}}\left[1-e^{-b z} \sum_{k=0}^{n-1} \frac{1}{k !}(b z)^{k}\right]
\end{gathered}
$$

where $n$ is a positive integer and $b>0$.

Following the development of (21), it is also possible to derive a closed-form expression for the average combined SNR by utilizing (25). Similarly, the ASEP and outage analyses can be carried out using this MGF, leading to a simpler result for positive integer $m$ case. These and more results will be discussed in our forthcoming work [13]

\section{APPROXIMATE ANALYSIS}

Although it is clear from (1) that $\gamma_{1}, \gamma_{2}, \ldots, \gamma_{14}$ are not independent random variables, but it is possible to derive a com- 
putationally efficient approximation for $\phi_{\gamma}($.$) by treating$ them to be independent. Hence, the development of $\phi_{\gamma}($.$) in$ this section rely on this assumption.

Suppose there are $L$ i.i.d diversity branches, it can be shown that the PDF of SNR of the $k$-th strongest diversity branch is given by

$$
\begin{aligned}
p_{k}(x) & =\frac{\partial}{\partial x} \sum_{i=0}^{k-1}\left(\begin{array}{c}
L \\
i
\end{array}\right)[F(x)]^{L-i}[1-F(x)]^{i} \\
& =k\left(\begin{array}{l}
L \\
k
\end{array}\right)_{w=0}^{L-k}(-1)^{w}\left(\begin{array}{c}
L-k \\
w
\end{array}\right)[1-F(x)]^{k-1+w} p(x) \\
& =k\left(\begin{array}{l}
L \\
k
\end{array}\right)_{w=0}^{k-1}(-1)^{w}\left(\begin{array}{c}
k-1 \\
w
\end{array}\right)[F(x)]^{L-k+w} p(x)
\end{aligned}
$$

The MGF of hybrid $M / L-S C / M R C$ combiner output SNR can therefore be approximated as

$$
\phi_{r}(s) \approx \prod_{k=1}^{M} \phi_{k}(s)
$$

by assuming $\gamma_{1}, \ldots, \gamma_{\mu}$ to be independent and $\phi_{k}($.$) repre-$ sents the MGF of $k$-th strongest diversity branch. Obviously, the approximation for $\phi_{y}($.$) collapses to the exact$ solution when $M=1$, as expected.

\section{A. Real Valued Fading Severity Index}

Substituting (3) and (4) into the third line of (30) and after simplifications, we get

$$
\begin{aligned}
p_{k}(x)= & k\left(\begin{array}{l}
L \\
k
\end{array}\right) \sum_{w=0}^{k-1}(-1)^{m}\left(\begin{array}{c}
k-1 \\
w
\end{array}\right)\left[\frac{(m x / \bar{\gamma})^{m}}{\Gamma(m)}\right]^{L-k+w+1} \\
& \times \exp \left(\frac{-m x}{\bar{\gamma}}\right) \sum_{n=0}^{\infty} c_{n} x^{n-1}
\end{aligned}
$$

where coefficients $c_{n}$ can be computed recursively and they are defined as in (5).

Taking the Laplace transform of the PDF (32) leads to

$$
\begin{aligned}
\phi_{k}(s) & =k\left(\begin{array}{l}
L \\
k
\end{array}\right) \sum_{w=0}^{k-1}(-1)^{w}\left(\begin{array}{c}
k-1 \\
w
\end{array}\right)\left[\frac{1}{\Gamma(m)}\left(\frac{m}{m+s \bar{\gamma}}\right)^{m}\right]^{L-k+w+1} \\
& \times \sum_{n=0}^{\infty} c_{n} \Gamma[n+m(L-k+w+1)]\left(\frac{\bar{\gamma}}{m+s \bar{\gamma}}\right)^{n}
\end{aligned}
$$

Finally, substituting (33) into (31) yields the desired MGF of the hybrid SC/MRC combiner output SNR. Notice that (31) holds for any order of $L$ and/or $M$, and all this are captured in a single formula.

\section{B. Positive Integer Fading Severity Index}

Similarly for positive integer $m$, it is straight-forward to show that the PDF and MGF of the $k$-th strongest branch are given by (34) and (35), respectively:

$$
\begin{gathered}
p_{k}(x)=\frac{k}{(m-1) !}\left(\begin{array}{l}
L \\
k
\end{array}\right)\left(\frac{m}{\bar{\gamma}}\right)^{L-k} \sum_{w=0}(-1)^{w}\left(\begin{array}{c}
L-k \\
w
\end{array}\right) \exp \left[-\frac{m x}{\bar{\gamma}}(k+w)\right] \\
\times \sum_{z=0}^{(m-1)(k-1+w)} \beta(z, k-1+w, m)\left(\frac{m x}{\bar{\gamma}}\right)^{z+m-1} \\
\phi_{k}(s)=\frac{k}{(m-1) !}\left(\begin{array}{l}
L \\
k
\end{array}\right) \sum_{w=0}^{L-k}(-1)^{w}\left(\begin{array}{c}
L-k \\
w
\end{array}\right) \\
\times \sum_{z=0}^{(m-1)(k-1+w)} \beta(z, k-1+w, m) \frac{(z+m-1) !}{[k+w+s \bar{\gamma} / m]^{z+m}}
\end{gathered}
$$

where coefficients $\beta(., . .$.$) may be computed using (24).$
For the special case of $M=1,(31)$ reduces to

$$
\phi_{y}(s)=\frac{L}{\Gamma(m)} \sum_{w=0}^{L-1}(-1)^{w}\left(\begin{array}{c}
L-1 \\
w
\end{array}\right)^{(m-1) w} \sum_{z=0}^{(m-1)} \frac{\beta(z, m) \Gamma(z+m)}{[1+w+s \bar{\gamma} / m]^{2+m}}
$$

which agrees with the result in [10] for $\mathrm{SC}$ receiver and (25) (in conjunction with (27)) as expected.

\section{CONCLUSIONS}

This paper derives new, concise expressions for the MGF and CDF of the hybrid SC/MRC combiner output SNR operating on Nakagami-m fading channels. A procedure for handling the case of arbitrary orders of $M$ and $L$ is also outlined. The MGF is used to facilitate an unified analysis of ASEP for different modulation schemes as well as to gain insights into the average combined SNR. Outage rate of error probability is computed using the CDF. An approximate solution for the MGF is also obtained by assuming that the ordered SNRs are independent. Finally, we point out that using the derivative formulas for ASEP derived in [14] along with the MGF expressions derived in this article, one can also analyze the performance of SC/EGC quadratic receivers without much difficulty.

\section{REFERENCES}

[1] T. Eng, N. Kong and L. B. Milstein, "Comparison of Diversity Combining Techniques for Rayleigh Fading Channels," IEEE Trans. on Communications, Vol. 44, pp. 1117-1129, Sept. 1996.

[2] K. J. Kim, S. Y. Kwon, E. K. Hong and K. C. Whang, "Comments on Comparison of Diversity Combining Techniques for Rayleigh Fading Channels" IEEE Trans. on Communications, Vol. 46, pp. 1109-1110 Sept. 1998.

[3] R. Wong, A. Annamalai and V. K. Bhargava, "Evaluation of Predetection Diversity Techniques for Rake Receivers," Proc. IEEE PACRIM'97, pp. 227-230, Aug. 1997.

[4] M. -S. Alouini and M. K. Simon, "Performance of Coherent Receivers with Hybrid SC/MRC over Nakagami-m Fading Channels," IEEE Trans. on Vehic. Technology, Vol. 48, pp. 1155-1164, July 1999.

[5] M. -S. Alouini and M. K. Simon, "Application of the Dirichlet Transformation to the Performance Evaluation of Generalized Selection Combining over Nakagami-m Fading Channels," Journal of Communications and Networks, Vol. 1, pp. 5-13, Mar. 1999.

[6] A. Annamalai, C. Tellambura and V. K. Bhargava, "Analysis of Hybrid Diversity Systems on Fading Channels," Proc. IEEE ISWC'99, pp. 70-71, June 1999.

[7] M. Win and J. Winters, "Analysis of Selection/Maximal-Ratio Combining in Rayleigh Fading," Proc. IEEE ICC'99, pp. 6-10, June 1999.

[8] A. Papoulis, Probability, Random Variables and Stochastic Processes, McGraw-Hill, New York: 1991.

[9] I. S. Gradshteyn and I. M. Ryzhik, Table of Integrals, Series and Products, Academic Press, 1995.

[10] A. Annamalai Jr. and C. Tellambura, "Error Rates for Nakagami-m Fading Multichannel Reception of Binary and M-ary Signals," to appear in the IEEE Trans. on Communications

[11] A. Annamalai Jr. and C. Tellambura, "Analysis of Hybrid Selection/Maximal-Ratio Diversity Combiners with Gaussian Errors," submitted to the IEEE Trans. on Communications.

[12] C. Tellambura and A. Annamalai, Tutorial Notes: Analytical Tools for Wireless Communications Systems Design, IEEE VTC'2000 (Spring).

[13] Annamalai and Tellambura, "Analysis of Hybrid SC/MRC Diversity Systems on Nakagami-m Channels," manuscript in preparation.

[14] A. Annamalai and C. Tellambura, "Incoherent Diversity Reception of M-ary Orthogonal Signals in Independent and Correlated Fading," submitted to the IEEE Trans. on Communications. 